\title{
Introduction to Part 1
}

In November 2005, the first in the series of International Conferences on High-Performance Embedded Architectures and Compilers (HiPEAC 2005) was held in Barcelona. We were fortunate to attract close to 100 submissions of which only 18 were selected for presentation. Among these, we asked the authors of the five most highly rated contributions to make extended versions of them. They all accepted to do that and their articles appear in this section of the volume.

The first article by Fursin et al. reports on an interesting approach to evaluate a large number of program optimizations. The aim is to self-tune a program against a specific architecture so as to achieve a shorter running time. In the second article by Geiger et al., the topic is energy-efficient cache design - also an important topic for embedded systems. They report on a multi-lateral cache design that is better tailored to the access characteristics of applications. In the third article by Buytaert et al., the topic is garbage collection - a performancecostly operation for object-oriented software systems. They propose a methodology by which garbage collection points, called hints, can be identified that reduces the cost of garbage collection. The fourth article, by Shi et al., addresses system designs that protect software confidentiality and integrity. To this end, they describe a new security model - MESA - that takes a memory-centric approach towards enhancing security in architectures. In the last article by Ning and Kaeli, the topic is energy-efficient bus design. They particularly address the power-inefficiency of bus arbitration algorithms and propose and evaluate novel algorithms that are shown to improve power-efficiency as well as performance.

Per Stenström

Chalmers University of Technology

Editor-in-chief Transactions on HiPEAC 